\title{
Intramolecular cyclization of $N$-(3-oxoalkenyl)phenylacetamides: synthesis of 3-phenyl-2(1H)-pyridones
}

\author{
Dmitry S. Goncharov, ${ }^{a}$ Anna K. Garkushenko, ${ }^{b}$ Alina P. Savelievab and Alexander S. \\ Fisyuk ${ }^{\text {b,c }}$. \\ ${ }^{a}$ Department of Pharmaceutical Chemistry, Omsk State Medical Academy, Lenin str., 12, \\ 644043 Omsk, Russian Federation \\ ${ }^{b}$ Department of Organic Chemistry, Omsk F.M. Dostoevsky State University, 55a Mira Ave, \\ 644077 Omsk, Russian Federation \\ ${ }^{c}$ Laboratory of New Organic Materials, Omsk State Technical University, 11 Mira Ave, 644050 \\ Omsk, Russian Federation \\ E-mail: fisyuk@,chemomsu.ru
}

DOI: http://dx.doi.org/10.3998/ark.5550190.p009.126

\begin{abstract}
3-Phenyl-2(1H)-pyridinones were obtained by base-catalyzed intramolecular aldol-type cyclization of $\mathrm{N}$-(3-oxoalkenyl)phenylacetamides. The effect of substituents in the starting compounds and the experimental conditions on the reaction course were established. It was shown that the transformations of $\mathrm{N}$-(3-oxoalkenyl)amides in basic medium depend on structural and electronic factors as well as the reaction conditions.
\end{abstract}

Keywords: 2(1H)-Pyridinone, $\beta$-enaminone, $N$-(3-oxoalkenyl)amide, intramolecular cyclization

\section{Introduction}

The 2(1H)-pyridinone ring is a structural fragment present within many alkaloids and other natural products. ${ }^{1-7}$ Further, a significant number of compounds with a $2(1 H)$-pyridinone motif exhibit interesting activities against a number of biological targets, and are used as scaffolds in drug discovery. ${ }^{8-11}$

Methods for 2(1H)-pyridinone ring formation can be based on bi- and multicomponent reactions, intramolecular cyclizations and transformation of other hyterocycles. ${ }^{12-16}$ While there are many ways of preparing 2-pyridinones, the study of new approaches to their synthesis is still desired due to the importance of this heterocycle.

Base-catalyzed intramolecular Knoevenagel condensation of bifunctional compounds 1-3, which contain in the molecule both carbonyl and amide groups, is one of the ways to construct a 
2-pyridinone ring. The cyclization of $o$-acylaminophenones $\mathbf{1}$ is well known as the Camps reaction and has been used for the synthesis of 2(1H)-quinolinones 4 and $4(1 H)$-quinolinones 5 since the $19^{\text {th }}$ century. ${ }^{17-22}$

The regioselectivity of the reaction depends on the $\mathrm{C}$ - $\mathrm{H}$ acidity at the $\alpha$-carbamoyl position of amides $\mathbf{1}$. Increasing the $\alpha$-carbamoyl acidity facilitates cyclization of amides $\mathbf{1}$ to $2(1 H)$ quinolinones 4 .

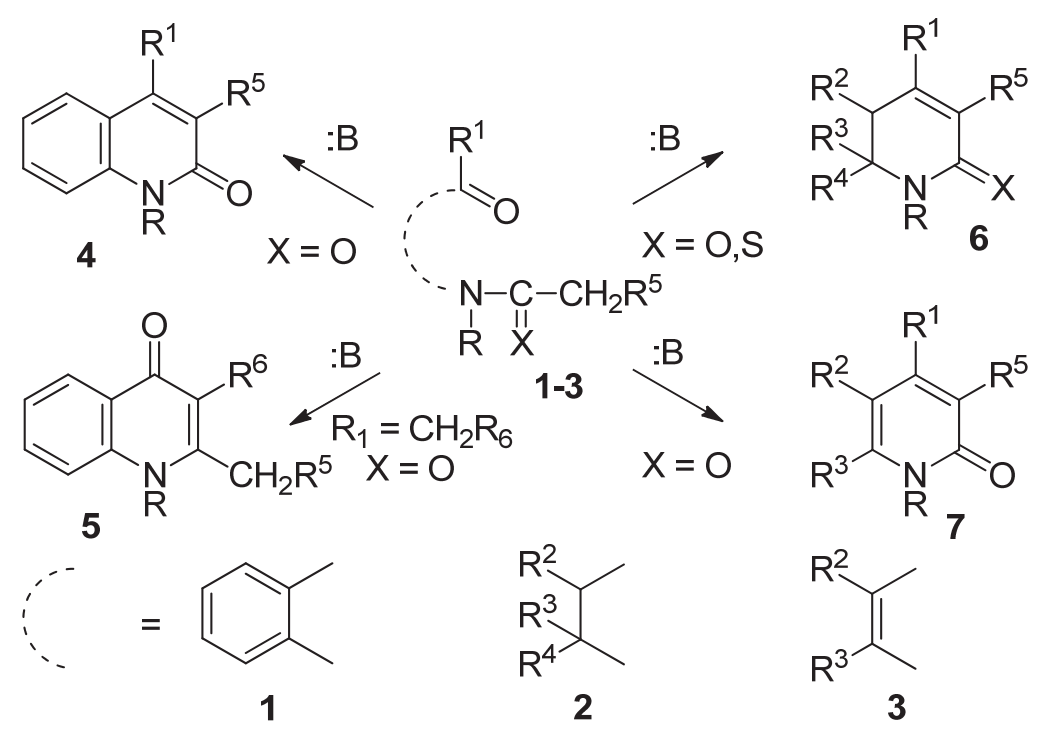

Scheme 1. Base-catalyzed intramolecular cyclization of $o$-acylaminophenones (1), $N-(3-$ oxoalkyl)amides (2) and $N$-(3-oxoalkenyl)amides (3).

$N$-(3-oxoalkyl)amides $\mathbf{2}$ and $N$-(3-oxoalkenyl)amides $\mathbf{3}$ are analogues of $O$ acylaminoacetophenones $\mathbf{1}$. A few years ago we developed the synthesis of 5,6-dihydro-2 $(1 \mathrm{H})$ pyridinones and -thiones $\mathbf{6}$ by intramolecular cyclization of $N$-(3-oxoalkyl)amides and thioamides $\mathbf{2}^{23-30}$ (Scheme 1). However, only a few examples of cyclization of $N$-(3oxoalkenyl)amides 3 to the $2(1 H)$-pyridinones 7 are known. ${ }^{31-36}$ Accordingly, the full synthetic potential of this reaction has not been studied.

In this paper we have widened the range of starting materials in order to elucidate the influence of both structural and electronic effects on this cyclization and, as a result, have determined its limitations.

\section{Results and Discussion}

Previously, we have reported that $N$-(3-oxoalkenyl) phenylacetamide $\mathbf{3 b}$ undergoes an aldol-type intramolecular ring closure to give 3-phenyl-2(1H)-pyridinone $\mathbf{7 b}{ }^{34}$ In order to establish the possibilities of this method we studied a similar cyclization for substituted $\mathrm{N}$-(3- 
oxoalkenyl)amides 3a-k. Compounds 3a-k were synthesized by acylation of enaminones 8a-k with phenylacetyl chloride in the presence of pyridine in $39-85 \%$ yields (Table 1).

Table 1. Preparation of $N$-(3-oxoalkenyl)amides 3a-k by N-acylation of enaminones 8a-k<smiles>[R]NC([R])=C([R])C([R])=O</smiles>

8a-k

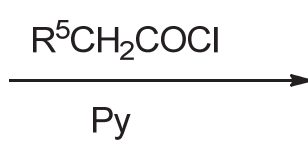<smiles>[R]C[R]</smiles>

\begin{tabular}{|c|c|c|c|c|c|c|c|}
\hline Entry & $\begin{array}{c}\text { Compou } \\
\text { nd }\end{array}$ & $\mathrm{R}^{1}$ & & $\mathrm{R}^{3}$ & $\mathrm{R}^{5}$ & $\mathrm{R}$ & Yield (\%) \\
\hline 1 & $Z-3 \mathbf{a}$ & $\mathrm{Me}$ & $\mathrm{H}$ & $\mathrm{Me}$ & $\mathrm{Ph}$ & $\mathrm{Me}$ & $45^{\mathrm{a}}$ \\
\hline 2 & $Z-\mathbf{3 b}$ & $\mathrm{Me}$ & $\mathrm{H}$ & $\mathrm{Me}$ & $\mathrm{Ph}$ & $\mathrm{H}$ & 56 \\
\hline 3 & $Z-3 c$ & $\mathrm{Me}$ & & $\left(\mathrm{CH}_{2}\right)_{4}$ & $\mathrm{Ph}$ & $\mathrm{H}$ & 78 \\
\hline 4 & $Z-3 d$ & $(\mathrm{Cl}$ & & $\mathrm{Me}$ & $\mathrm{Ph}$ & $\mathrm{H}$ & 67 \\
\hline 5 & $Z-3 \mathbf{e}$ & $\mathrm{Ph}$ & $\mathrm{H}$ & $\mathrm{Me}$ & $\mathrm{Ph}$ & $\mathrm{H}$ & 85 \\
\hline 6 & $Z-\mathbf{3 f}$ & $\mathrm{C}_{6} \mathrm{H}_{4}-4-\mathrm{Me}$ & $\mathrm{H}$ & $\mathrm{Me}$ & $\mathrm{Ph}$ & $\mathrm{H}$ & 70 \\
\hline 7 & $Z-3 g$ & $\mathrm{C}_{6} \mathrm{H}_{4}-4-\mathrm{Cl}$ & $\mathrm{H}$ & $\mathrm{Me}$ & $\mathrm{Ph}$ & $\mathrm{H}$ & 65 \\
\hline 8 & $Z-3 \mathbf{h}$ & 2-Naphthyl & $\mathrm{H}$ & $\mathrm{Me}$ & $\mathrm{Ph}$ & $\mathrm{H}$ & 70 \\
\hline 9 & $Z-3 \mathbf{i}$ & 1-Naphthyl & $\mathrm{H}$ & $\mathrm{Me}$ & $\mathrm{Ph}$ & $\mathrm{H}$ & 63 \\
\hline 10 & $Z-3 \mathbf{j}$ & $\mathrm{Me}$ & $\mathrm{H}$ & $\mathrm{Me}$ & $\mathrm{H}$ & $\mathrm{H}$ & 74 \\
\hline 11 & $E-, Z-3 \mathbf{k}$ & $\left(\mathrm{CH}_{2}\right)_{4}$ & & $\mathrm{H}$ & $\mathrm{Ph}$ & $\mathrm{H}$ & 39 \\
\hline
\end{tabular}

${ }^{\mathrm{a}}$ Yield of the isolated product after column chromatography

It was found that cyclization of compound Z-3a occurs at room temperature over 15 min by the action of potassium hydroxide in ethanol to give the 2-pyridinone $7 \mathbf{a}$ in 94\% yield (Table 2). On the other hand, the cyclization of secondary amide $\mathbf{3 b}$ did not occur under the action of an alcoholic solution of alkali. According to TLC analysis, enaminone $\mathbf{8 b}$, the product of hydrolysis of $N$-(3-oxoalkenyl)amide $\mathbf{3 b}$, was identified in the reaction mixture. 3-Phenyl-2(1H)pyridinones $7 \mathbf{b}$-i were obtained only in dry THF using 1.5 equivalents of potassium tert-butoxide and a cyclization time of 5-18 hours with $40-73 \%$ yields. However, in this case too, according to chromato-mass spectrometry data, enaminones 8a-i were present in the reaction media. The appearance of enaminones 8a-i as by-products was a result of hydrolysis of compounds 3a-i by the water which was formed in the cyclization process.

Attempts to carry out cyclization of $\mathrm{N}$-(3-oxoalkyl)acetamide $\mathbf{3 j}$ which has low $\mathrm{C}-\mathrm{H}$ acidity at the $\alpha$-carbamoyl position, in basic conditions were unsuccessful. Secondary amide $\mathbf{3 j}$ remained unchanged even on heating with potassium tert-butoxide. Apparently only $N$-(3- 
oxoalkenyl)amides having significant $\mathrm{C}$-H acidity at the $\alpha$-carbamoyl position, are capable of cyclisation via an aldol condensation pathway in basic conditions. Increasing the $\alpha$-carbamoyl acidity facilitates cyclization, while its low acidity makes cyclization impossible.

Thus the cyclization of the tertiary amide Z-3a proceeds under milder conditions and with better yield in comparison with secondary amides $\mathbf{3 b}$-i. In contrast to the tertiary amide Z-3a, the cyclization of secondary amides $\mathbf{3} \mathbf{b}$-i to $2(1 H)$-pyridinones $\mathbf{7 b}$-i occurs via formation of dianions $Z$-10b-i. Deprotonation of N-hydrogen in the first stage of interaction of secondary amides $\mathbf{3 b} \mathbf{b}-\mathbf{i}$ with potassium tert-butoxide leads to formation of mesomeric anions $\mathbf{9 b}$-i, which have a delocalized negative charge in an $\beta$-enaminoketone fragment. As a result of the charge delocalization, the carbonyl group activity of anions $\mathbf{9 b - i}$ decreases. This leads to a decrease of the cyclization rate of secondary amides $\mathbf{3 b} \mathbf{b} \mathbf{i}$ in comparison with the tertiary example $\mathbf{3} \mathbf{a}$.

Table 2. Scope of the cyclization $N$-(3-oxoalkenyl)phenylacetamide 3 to synthesize 3-phenyl-2(1H)-pyridinones 7

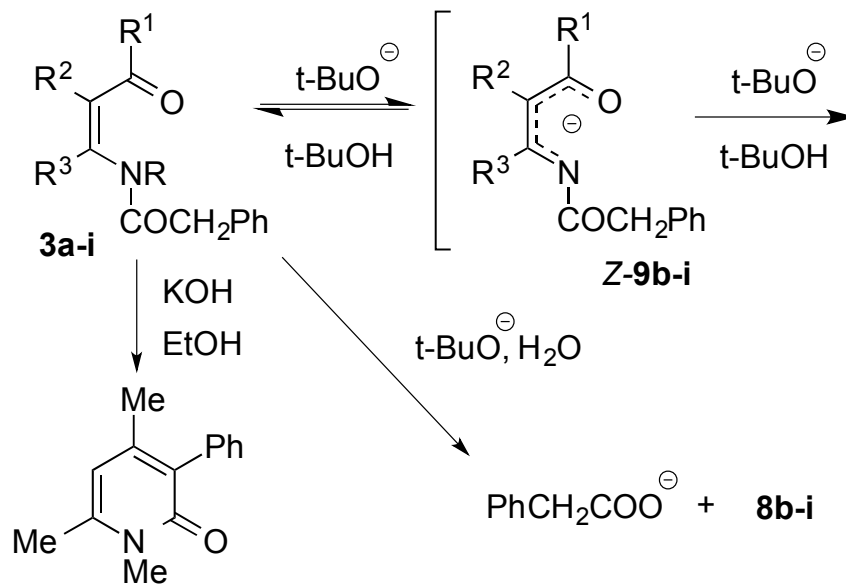

$7 a$

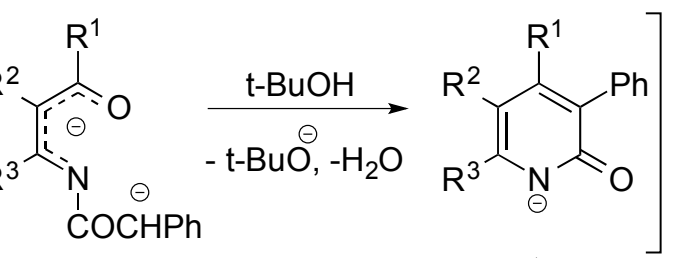

Z-10b-i

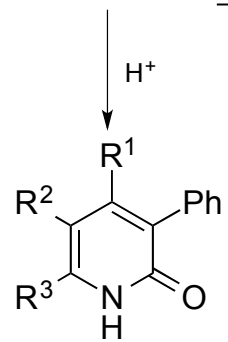

7b-i

Entry Product $\begin{gathered}\text { Yield Entry } \\ (\%)\end{gathered}$


Table 2. Continued

Entry Product $\begin{array}{c}\text { Yield Entry } \\ (\%)\end{array}$ Product $\left.\begin{array}{c}\text { Yield } \\ (\%)\end{array}\right)$

The NH signals of $N$-(3-oxoalkenyl)amides $\mathbf{3 b}$-j are present in the low field region $(\delta(\mathrm{NH}) \sim 12)$ in the ${ }^{1} \mathrm{H}$ NMR spectra. This suggests a strong intramolecular hydrogen bonding between the amino group and the carbonyl oxygen. These compounds exist in the $Z-S-Z$ form in $\mathrm{CDCl}_{3}$ solution. ${ }^{37,38}$ In contrast to compounds $\mathbf{3 b} \mathbf{b} \mathbf{j}$, a mixture of two isomers $Z$ - and $E$-3k was obtained. $Z$ - and $E$-isomers $3 \mathbf{k}$ were separated by column chromatography on silica gel (Scheme $2)$. The isomer $Z$-3k had a broad NH signal at low field $(\delta(\mathrm{NH})=11.32)$, but the NH signal of $E$ isomer 3k was a doublet with a coupling constant ${ }^{3} J_{\mathrm{NHCH}}=12.1 \mathrm{~Hz}$ and chemical shift $7.13 \mathrm{ppm}$. A broad absorbance of the NH of compound Z-3k was at $3200-3600 \mathrm{~cm}^{-1}$ in its IR spectrum. At the same time, the $\mathrm{NH}$ bond $E$-isomer $3 \mathbf{k}$ is fixed as a narrow peak at $3246 \mathrm{~cm}^{-1}$. According to ${ }^{1} \mathrm{H}$ NMR analysis, transformation of $Z$-isomer $3 \mathbf{k}$ into a mixture of the $Z$ - and $E$-isomers in the 6:1 ratio in solution of $\mathrm{CDCl}_{3}$ takes two days. The addition of tetramethylguanidine led to rapid isomerization of compound 3k. Z-Isomers 3k are stabilized by an intramolecular hydrogen bond, which disappears after deprotonation of nitrogen atom. The nature of substituents influences both the charge distribution in mesomeric anion 9 and the stability of $Z$ - and $E$-isomers. Alkyl groups stabilize a double bond. Therefore the double bond of anion Z-9k $\left(\mathrm{R}^{3}=\mathrm{H}\right)$ is more stable at the position $\mathrm{C}(2), \mathrm{C}(3)$ (Scheme 2$)$ in comparison with anions $9 \mathbf{b}-\mathbf{i}\left(\mathrm{R}^{3}=\mathrm{Alk}\right)$ which have a more stable double bond at the position $\mathrm{C}(1), \mathrm{C}(2)$ (Table 2 ). The stability of $Z$-and $E$-isomers 3 depends on the size of the substituents in the 3-oxoalkenyl fragment. Increasing the size of substituent $\mathrm{R}^{3}$ should lead to a destabilization of the $E$-isomers of deprotonated $N$-(3oxoalkenyl)amides $\mathbf{3}$ due to steric interactions with the carbonyl group, and vice versa - a decrease in the size of $\mathrm{R}^{3}$ should stabilize the $E$-isomers, like the enaminones. ${ }^{39-43}$ Thus the charge distribution in mesomeric anion 9 as well as the isomerization $Z-3 \mathbf{k}$ to $E$-3k prevent the intramolecular ring closure of $N$-(3-oxoalkenyl)amide $3 \mathbf{k}$ to a corresponding $2(1 H)$-pyridinone. Obviously, for this reason the cyclization of amides Z-3k does not occur under the action of potassium tert-butoxide in THF. In this case, the reaction product was a mixture of $Z$ - and $E$ isomers $3 \mathbf{k}$. 


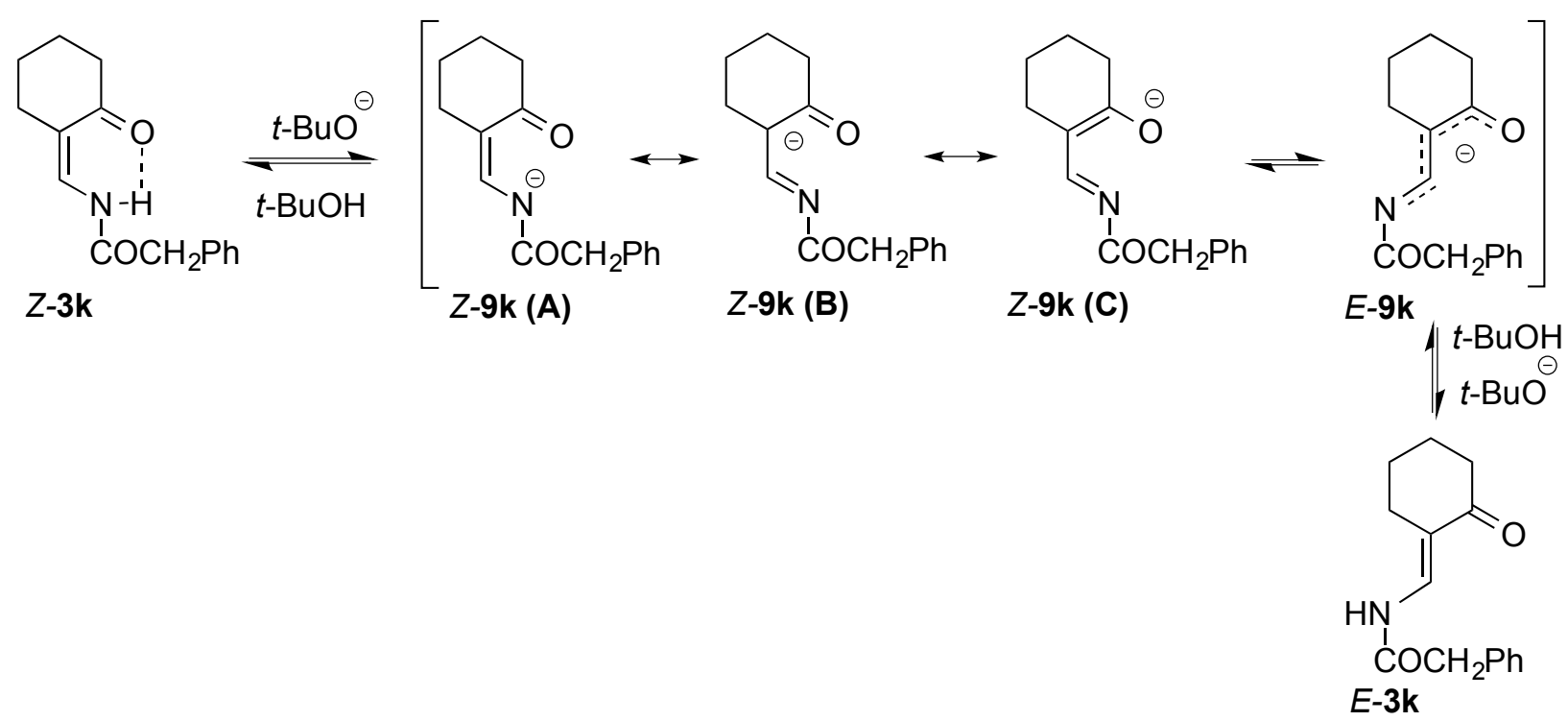

Scheme 2. Interaction of $N$-(3-oxoalkenyl)phenylacetamide $3 \mathbf{k}$ with potassium $t$-butoxide.

\section{Conclusions}

The intramolecular aldol-type condensation of bifunctional compounds containing carbonyl and amide groups is a general method for the synthesis of $2(1 H)$-pyridinones and derivatives. This approach can be successfully used for the preparation of $2(1 H)$-quinolinones, 5,6-dihydro- $2(1 H)$ pyridinones. The transformations of $N$-(3-oxoalkenyl)amides in basic medium depend on structural, electronic factors and the reaction conditions. However, availability and diversity of starting materials and a simple experimental procedure make the $N$ - $(3-$ oxoalkenyl)phenylacetamides convenient precursors for the synthesis of 3-phenyl-2(1H)pyridinones.

\section{Experimental Section}

General. The ${ }^{1} \mathrm{H}$ and ${ }^{13} \mathrm{C}$ NMR spectra were recorded on a Bruker ARX-300 or a Bruker DRX400 instruments, with TMS as internal standard. The IR spectra were recorded on a INFRALUM FT-801 spectrometer. The mass spectra were recorded on an Agilent 6890 gas chromatograph coupled with a $5973 \mathrm{~N}$ quadrupole mass-selective electron impact (EI) detector or the Thermo Scientific DSQ II GC/MS with TRACE GC Ultra (70 eV, evaporator temperature 200-250 ${ }^{\circ} \mathrm{C}$ ). The reaction course and purity of the products were checked by thin-layer chromatography on Sorbfil UV-254 plates. Compounds $8 \mathbf{8},{ }^{39} \mathbf{8 b},{ }^{40} \mathbf{8 c}, \mathbf{d},{ }^{41} \mathbf{8 e},{ }^{40} \mathbf{8 j},{ }^{42} \mathbf{8} \mathbf{k}^{43}$ and $\mathbf{3 j}{ }^{37}$ were prepared as previously reported. The physical constants and spectral data of the compounds $\mathbf{3 b}, \mathbf{7 b}$ were given earlier by us. ${ }^{34}$ Copies of NMR spectra are given in the supporting information. 
General procedures for the synthesis of enaminones (8e-i). A slow stream of ammonia gas was passed through a solution of the relevant 1,3 -diketone $(0.1 \mathrm{~mol})$ in dry toluene $(20 \mathrm{~mL})$ and a catalytic amount of formic acid. The mixture was heated under reflux and the $\mathrm{H}_{2} \mathrm{O}$ formed was removed azeotropically using a Dean-Stark apparatus. After cooling the reaction mixture a precipitate of enaminone was filtered off and recrystallized.

3-Amino-1-(4-methylphenyl)but-2-en-1-one (8f).Yield 85\%, light crystals, mp 89-90 ${ }^{\circ} \mathrm{C}$ (toluene) (lit. $\left.{ }^{41} \mathrm{mp} \mathrm{89-90}{ }^{\circ} \mathrm{C}\right) . \mathrm{IR}(\mathrm{KBr}): v, \mathrm{~cm}^{-1} 3297,3143\left(\mathrm{NH}_{2}\right), 1602(\mathrm{C}=\mathrm{O}), 1535(\mathrm{C}=\mathrm{C}) .{ }^{1} \mathrm{H}$ NMR (400 MHz, $\left.\mathrm{CDCl}_{3}\right): \delta 2.02\left(3 \mathrm{H}, \mathrm{s}, \mathrm{CH}_{3}\right), 2.37\left(3 \mathrm{H}, \mathrm{s}, 4-\mathrm{CH}_{3}\right), 5.39\left(1 \mathrm{H}\right.$, br.s, $\left.\mathrm{NH}_{2}\right), 5.71$ $(1 \mathrm{H}, \mathrm{s},-\mathrm{CH}=), 7.17-7.23(2 \mathrm{H}, \mathrm{m}, \mathrm{H} \mathrm{Ar}), 7.75-7.81(2 \mathrm{H}, \mathrm{m}, \mathrm{H} \mathrm{Ar}), 10.16\left(1 \mathrm{H}, \mathrm{br} . \mathrm{s}, \mathrm{NH}_{2}\right) .{ }^{13} \mathrm{C}$ NMR (100 MHz, $\left.\mathrm{CDCl}_{3}\right): \delta 21.4\left(4-\mathrm{CH}_{3}\right), 22.8\left(=\mathrm{C}-\underline{\mathrm{CH}}_{3}\right), 92.1(=\mathrm{CH}-), 127.2,128.9,137.6$, 141.1 (Ar), 162.8 (=ㄷ- $\left.\mathrm{CH}_{3}\right), 189.3$ (을).

3-Amino-1-(4-chlorophenyl)but-2-en-1-one (8g). Yield 75\%, light crystals, mp 128-129 ${ }^{\circ} \mathrm{C}$ (toluene) (lit. $\left.{ }^{42} \mathrm{mp} 128-129^{\circ} \mathrm{C}\right)$. IR (KBr): $v$ cm $^{-1}$ 3285, $3146\left(\mathrm{NH}_{2}\right), 1603(\mathrm{C}=\mathrm{O}), 1529(\mathrm{C}=\mathrm{C})$. ${ }^{1} \mathrm{H}$ NMR (400 MHz, $\left.\mathrm{CDCl}_{3}\right): \delta 2.04\left(3 \mathrm{H}, \mathrm{s}, \mathrm{CH}_{3}\right), 5.40\left(1 \mathrm{H}\right.$, br.s, $\left.\mathrm{NH}_{2}\right), 5.66(1 \mathrm{H}, \mathrm{s},-\mathrm{CH}=)$, 7.33-7.39 (2H, m, H Ar), 7.77-7.83 (2H, m, H Ar), 10.20 (1H, br.s, $\left.\mathrm{NH}_{2}\right) .{ }^{13} \mathrm{C} \mathrm{NMR} \mathrm{(100} \mathrm{MHz,}$ $\left.\mathrm{CDCl}_{3}\right): \delta 22.8\left(=\mathrm{C}-\underline{\mathrm{CH}}_{3}\right), 92.0(=\mathrm{CH}-), 128.4,128.5,136.9,138.6(\mathrm{Ar}), 163.5\left(=\underline{\mathrm{C}}_{-} \mathrm{CH}_{3}\right), 187.9$ (COAr); Anal. Calcd for: $\mathrm{C}_{10} \mathrm{H}_{10} \mathrm{ClNO}$ C, 61.39; H, 5.15; N, 7.16. Found: C, 61.42; H, 5.14; N, $7.20 \%$.

3-Amino-1-(2-naphthyl)but-2-en-1-one (8h). Yield 83\%, light crystals, mp 148-149 ${ }^{\circ} \mathrm{C}$ (toluene); IR (KBr): $v, \mathrm{~cm}^{-1}$ 3293, $3138\left(\mathrm{NH}_{2}\right), 1612(\mathrm{C}=\mathrm{O}), 1530(\mathrm{C}=\mathrm{C}) .{ }^{1} \mathrm{H}$ NMR $(400 \mathrm{MHz}$, $\left.\mathrm{CDCl}_{3}\right): \delta 2.09\left(3 \mathrm{H}, \mathrm{s}, \mathrm{CH}_{3}\right), 5.25\left(1 \mathrm{H}\right.$, br.s, $\left.\mathrm{NH}_{2}\right), 5.90(1 \mathrm{H}, \mathrm{s},-\mathrm{CH}=), 7.47-7.55(2 \mathrm{H}, \mathrm{m}, \mathrm{H} \mathrm{Ar})$, 7.83-7.89 (2H, m, H Ar), 7.91-7.96 (1H, m, H Ar), 7.98-8.02 (1H, m, H Ar), 8.39 (1H, s, H-1' Ar), $10.28\left(1 \mathrm{H}\right.$, br.s, $\left.\mathrm{NH}_{2}\right) .{ }^{13} \mathrm{C}$ NMR $\left(100 \mathrm{MHz}, \mathrm{CDCl}_{3}\right): \delta 22.8\left(=\mathrm{C}-\mathrm{CH}_{3}\right), 92.6(=\mathrm{CH}-), 124.2$, 126.2 , 127.1, 127.5, 127.6, 127.9, 129.2, 132.9, 134.7, 137.6 (Ar), $162.8\left(=\underline{\mathrm{C}}-\mathrm{CH}_{3}\right), 189.3$ (OAA); Anal. Calcd for: $\mathrm{C}_{14} \mathrm{H}_{13} \mathrm{NO}$ C, 79.59; H, 6.20; N, 6.63. Found: C, 79.53; H, 6.21; N, $6.59 \%$.

3-Amino-1-(1-naphthyl)but-2-en-1-one (8i). Yield 87\%, light crystals, mp 159-160 ${ }^{\circ} \mathrm{C}$ (toluene); IR (KBr): $v, \mathrm{~cm}^{-1}$ 3287, $3133\left(\mathrm{NH}_{2}\right), 1617(\mathrm{C}=\mathrm{O}), 1524(\mathrm{C}=\mathrm{C}) .{ }^{1} \mathrm{H}$ NMR (400 MHz, $\left.\mathrm{CDCl}_{3}\right): \delta 1.98\left(3 \mathrm{H}, \mathrm{s}, \mathrm{CH}_{3}\right), 5.46\left(1 \mathrm{H}\right.$, br.s, $\left.\mathrm{NH}_{2}\right), 5.50(1 \mathrm{H}, \mathrm{s},-\mathrm{CH}=), 7.43-7.54(3 \mathrm{H}, \mathrm{m}, \mathrm{H} \mathrm{Ar})$, 7.61-7.65 (1H, m, H Ar), 7.83-7.88 (2H, m, H Ar), 8.41-8.45 (1H, m, H Ar), 10.22 (1H, br.s, $\left.\mathrm{NH}_{2}\right) .{ }^{13} \mathrm{C}$ NMR $\left(100 \mathrm{MHz}, \mathrm{CDCl}_{3}\right): \delta 22.6\left(=\mathrm{C}-\mathrm{CH}_{3}\right), 97.0(=\mathrm{CH}-), 124.8,125.1,125.9,126.1$,

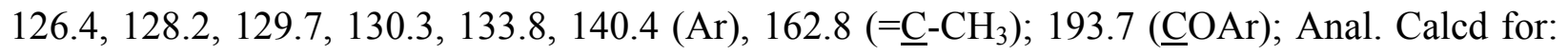
$\mathrm{C}_{14} \mathrm{H}_{13} \mathrm{NO}$ C, 79.59; H, 6.20; N, 6.63. Found: C, 79.59; H, 6.17; N, 6.64\%.

General procedure for the synthesis of $\boldsymbol{N}$-(3-oxoalkenyl)phenylacetamides (3a-k). Phenylacetyl chloride $1.623 \mathrm{~g}(10.5 \mathrm{mmol})$ was added dropwise to a solution of enamino ketone $(10.0 \mathrm{mmol})$ and anhydrous pyridine $(1 \mathrm{~mL})$ in absolute $\mathrm{CHCl}_{3}(15 \mathrm{~mL})$ with stirring. The mixture was stirred for $1 \mathrm{~h}$ with cooling in ice and for 4-10 $\mathrm{h}$ at room temperature. $\mathrm{CHCl}_{3}(10$ $\mathrm{mL}$ ) was then added and the reaction mixture was washed with $10 \%$ aq $\mathrm{HCl}$ solution $(30 \mathrm{~mL})$ and with $\mathrm{H}_{2} \mathrm{O}$ until the wash water gave a neutral reaction. The organic phase was dried with 
anhydrous $\mathrm{Na}_{2} \mathrm{SO}_{4}$, and the $\mathrm{CHCl}_{3}$ distilled off. The compound was recrystallized from an EtOAc-petroleum ester mixture $4 / 7 \quad(\mathbf{3 b}, \mathbf{h}, \mathbf{i}, \mathbf{j}), \quad i-\mathrm{PrOH} \quad(\mathbf{3 f}, \mathbf{g})$ or purified by column chromatography $\left(\mathrm{Al}_{2} \mathrm{O}_{3}, \mathrm{CH}_{2} \mathrm{Cl}_{2}\right.$-hexane (3c-e), hexane-EtOAc 5/1 (Z-3a) or on silica gel $\mathrm{L}$ 40/100, $\mathrm{CHCl}_{3}-\mathrm{EtOAc} 1 / 1(E, Z-3 \mathbf{k})$.

$N$-methyl- $N$-[(Z)-1-methyl-3-oxo-but-1-enyl]-2-phenylacetamide (Z-3a). Yield $45 \%$, light yellow oil; IR (KBr): $v, \mathrm{~cm}^{-1} 1693(\mathrm{C}=\mathrm{O}), 1612(\mathrm{~N}-\mathrm{C}=\mathrm{O}) .{ }^{1} \mathrm{H}$ NMR $\left(400 \mathrm{MHz}, \mathrm{CDCl}_{3}\right): \delta 2.14$ $\left(3 \mathrm{H}, \mathrm{s}, \mathrm{COCH}_{3}\right), 2.22\left(3 \mathrm{H}, \mathrm{s},=\mathrm{C}_{-} \mathrm{CH}_{3}\right), 3.06\left(3 \mathrm{H}, \mathrm{s}, \mathrm{N}-\mathrm{CH}_{3}\right), 3.70\left(2 \mathrm{H}, \mathrm{s}, \mathrm{CH}_{2}\right), 5.97(1 \mathrm{H}, \mathrm{s}$, $=\mathrm{CH}), 7.20-7.35\left(5 \mathrm{H}, \mathrm{m}, \mathrm{C}_{6} \mathrm{H}_{5}\right) .{ }^{13} \mathrm{C}$ NMR $\left(100 \mathrm{MHz}, \mathrm{CDCl}_{3}\right): \delta 18.5\left(=\mathrm{C}-\mathrm{CH}_{3}\right), 32.0\left(\mathrm{~N}_{-}-\mathrm{CH}_{3}\right)$, $34.2\left(\mathrm{COCH}_{3}\right), 41.4\left(\mathrm{C}_{6} \mathrm{H}_{5}-\underline{\mathrm{CH}}_{2}\right), 124.7$ (= $\left.\mathrm{CHCO}\right), 126.9\left(\mathrm{C}_{6} \mathrm{H}_{5}, \mathrm{C}-4^{\prime}\right), 128.7\left(\mathrm{C}_{6} \mathrm{H}_{5}, \mathrm{C}-2^{\prime}, \mathrm{C}-6^{\prime}\right)$, $128.8\left(\mathrm{C}_{6} \mathrm{H}_{5}, \mathrm{C}-3^{\prime}, \mathrm{C}^{2} 5^{\prime}\right), 135.3\left(\mathrm{C}_{6} \mathrm{H}_{5}, \mathrm{C}-1^{\prime}\right), 154.2(=\mathrm{C}-\mathrm{N}), 169.8(\mathrm{NHCO}), 197.7(\mathrm{C}=\mathrm{O})$; Anal. Calcd for: $\mathrm{C}_{14} \mathrm{H}_{17} \mathrm{NO}_{2} \mathrm{C}, 72.70 ; \mathrm{H}, 7.41 ; \mathrm{N}, 6.06$. Found: $\mathrm{C}, 72.77 ; \mathrm{H}, 7.46 ; \mathrm{N}, 6.12 \%$.

$N$-I(Z)-2-acetylcyclohex-1-enyl]-2-phenylacetamide $(Z-3 c)$. Yield 78\%, light yellow oil; IR $\left(\mathrm{CHCl}_{3}\right): v, \mathrm{~cm}^{-1}$ 3250-3150 (NH), $1697(\mathrm{C}=\mathrm{O}), 1632(\mathrm{C}=\mathrm{O}), 1585(\mathrm{~N}-\mathrm{C}=\mathrm{O}) .{ }^{1} \mathrm{H}$ NMR $(400$ $\left.\mathrm{MHz}, \mathrm{CDCl}_{3}\right): \delta 1.54-1.66(4 \mathrm{H}, \mathrm{m}, 2 \mathrm{H}-4,2 \mathrm{H}-5), 2.17\left(3 \mathrm{H}, \mathrm{s}, \mathrm{CH}_{3}\right), 2.31-2.39(2 \mathrm{H}, \mathrm{m}, 2 \mathrm{H}-3)$, 2.91-3.03 (2H, m, 2H-2), $3.61\left(2 \mathrm{H}, \mathrm{s}, \mathrm{CH}_{2}\right), 5.30(1 \mathrm{H}, \mathrm{s},=\mathrm{CH}), 7.22-7.29\left(5 \mathrm{H}, \mathrm{m}, \mathrm{C}_{6} \mathrm{H}_{5}\right), 12.86$ (1H, br. s, NH). ${ }^{13} \mathrm{C}$ NMR $\left(100 \mathrm{MHz}, \mathrm{CDCl}_{3}\right): \delta 21.5\left(\mathrm{CH}_{2}\right), 22.0\left(\mathrm{CH}_{2}\right), 26.0\left(\mathrm{CH}_{2}\right), 28.6\left(\mathrm{CH}_{2}\right)$, $29.0\left(\mathrm{CH}_{3}\right) ; 46.1\left(\mathrm{C}_{6} \mathrm{H}_{5}-\underline{\mathrm{C}_{2}}\right) ; 111.8(=\underline{\mathrm{CCO}}) ; 127.2\left(\mathrm{C}_{6} \mathrm{H}_{5}, \mathrm{C}-{ }^{\prime}\right), 128.8\left(\mathrm{C}_{6} \mathrm{H}_{5}, \mathrm{C}-2^{\prime}, \mathrm{C}-6^{\prime}\right), 129.4$ $\left(\mathrm{C}_{6} \mathrm{H}_{5}, \mathrm{C}-3^{\prime}, \mathrm{C}-5^{\prime}\right), 133.4\left(\mathrm{C}_{6} \mathrm{H}_{5}, \mathrm{C}-1^{\prime}\right), 152.6(=\mathrm{C}-\mathrm{N}), 170.5(\mathrm{NHCO}), 202.5(\mathrm{C}=\mathrm{O})$; Anal. Calcd for: $\mathrm{C}_{16} \mathrm{H}_{19} \mathrm{NO}_{2} \mathrm{C}, 74.68 ; \mathrm{H}, 7.44 ; \mathrm{N}$, 5.44. Found: $\mathrm{C}, 74.73 ; \mathrm{H}, 7.36 ; \mathrm{N}, 5.35 \%$.

$\mathrm{N}$-\{1-[2-oxocyclopent-(Z)-ylidene]-ethyl\}-2-phenylacetamide (Z-3d). Yield 67\%, light yellow crystals, mp 56-57 ${ }^{\circ} \mathrm{C}$. IR (KBr): $v, \mathrm{~cm}^{-1} 3250-3150(\mathrm{NH}), 1717(\mathrm{C}=\mathrm{O}), 1683(\mathrm{C}=\mathrm{O}), 1615(\mathrm{~N}-$ $\mathrm{C}=\mathrm{O} .{ }^{1} \mathrm{H}$ NMR $\left(400 \mathrm{MHz}, \mathrm{CDCl}_{3}\right): \delta 1.79-1.97(2 \mathrm{H}, \mathrm{m}, 2 \mathrm{H}-4), 2.24-2.48\left(5 \mathrm{H}, \mathrm{m}, 2 \mathrm{H}-5, \mathrm{CH}_{3}\right)$, 2.49-2.66 (2H, m, 2H-3), $3.66\left(2 \mathrm{H}, \mathrm{s}, \mathrm{CH}_{2}\right), 7.22-7.47\left(5 \mathrm{H}, \mathrm{m}, \mathrm{C}_{6} \mathrm{H}_{5}\right), 12.01(1 \mathrm{H}$, br.s, $\mathrm{NH}) .{ }^{13} \mathrm{C}$ NMR (100 MHz, CDCl 3$): \delta 18.5\left(\mathrm{CH}_{3}\right), 19.6\left(\mathrm{CH}_{2}\right), 27.2\left(\mathrm{CH}_{2}\right), 39.7\left(\mathrm{CH}_{2}\right), 45.6\left(\mathrm{C}_{6} \mathrm{H}_{5}-\mathrm{CH}_{2}\right)$, $114.5(=\underline{C C O}), 127.3\left(\mathrm{C}_{6} \mathrm{H}_{5}, \mathrm{C}-4^{\prime}\right), 128.8\left(\mathrm{C}_{6} \mathrm{H}_{5}, \mathrm{C}-2^{\prime}, \mathrm{C}-6^{\prime}\right), 129.4\left(\mathrm{C}_{6} \mathrm{H}_{5}, \mathrm{C}-3^{\prime}, \mathrm{C}-5^{\prime}\right), 134.0\left(\mathrm{C}_{6} \mathrm{H}_{5}\right.$, C-1'), $148.1(=\mathrm{C}-\mathrm{N}), 170.4(\mathrm{NHCO}), 207.8(\mathrm{C}=\mathrm{O})$; Anal. Calcd for: $\mathrm{C}_{15} \mathrm{H}_{17} \mathrm{NO}_{2} \mathrm{C}, 74.05 ; \mathrm{H}$, 7.04; N, 5.76. Found: C, 74.12; H, 7.07; N, 5.81\%.

$N$-[(Z)-1-methyl-3-oxo-3-phenylprop-1-en-1-yl]-2-phenylacetamide $(Z-3 e)$. Yield $85 \%$, white crystals, mp 75-76 ${ }^{\circ} \mathrm{C}$. IR $(\mathrm{KBr}): v, \mathrm{~cm}^{-1} 3250-3150(\mathrm{NH}), 1702(\mathrm{C}=\mathrm{O}), 1624(\mathrm{C}=\mathrm{O}), 1591(\mathrm{~N}-$ $\mathrm{C}=\mathrm{O}) .{ }^{1} \mathrm{H}$ NMR $\left(400 \mathrm{MHz}, \mathrm{CDCl}_{3}\right): \delta 2.49\left(3 \mathrm{H}, \mathrm{d}, J 0.9 \mathrm{~Hz}, \mathrm{CH}_{3}\right), 3.72\left(2 \mathrm{H}, \mathrm{s}, \mathrm{CH}_{2}\right), 6.02(1 \mathrm{H}$, d, $J 0.9 \mathrm{~Hz}, \mathrm{CH}=), 7.26-7.55(5 \mathrm{H}, \mathrm{m}, \mathrm{Ar}-\mathrm{H}), 7.84-7.91(5 \mathrm{H}, \mathrm{m}, \mathrm{Ar}-\mathrm{H}), 12.88(1 \mathrm{H}$, br. s, NH). ${ }^{13} \mathrm{C}$ NMR $\left(100 \mathrm{MHz}, \mathrm{CDCl}_{3}\right): \delta 22.4\left(=\mathrm{C}-\underline{\mathrm{CH}}_{3}\right) ; 45.6\left(\mathrm{COCH}_{2}\right) ; 102.1(=\mathrm{CH}), 127.3,127.6$, 128.5, 128.8, 129.4, 132.3, 133.8, 138.7 (Ar), 157.3 (=C-N), 170.7 (NHCO), 191.4 (COAr); Anal. Calcd for: $\mathrm{C}_{18} \mathrm{H}_{17} \mathrm{NO}_{2} \mathrm{C}, 77.40 ; \mathrm{H}, 6.13 ; \mathrm{N}, 5.01$. Found: C, 77.48; H, 6.18; N, 5.07\%.

$\mathrm{N}$-[(Z)-1-methyl-3-(4-methylphenyl)-3-oxoprop-1-en-1-yl]-2-phenylacetamide (Z-3f). Yield $70 \%$, white crystals, mp 104-105. ${ }^{\circ} \mathrm{C} . \mathrm{IR}(\mathrm{KBr}): v, \mathrm{~cm}^{-1} 3463(\mathrm{NH}), 1703(\mathrm{C}=\mathrm{O}), 1607(\mathrm{C}=\mathrm{O})$, $1588(\mathrm{~N}-\mathrm{C}=\mathrm{O}) .{ }^{1} \mathrm{H}$ NMR $\left(400 \mathrm{MHz}, \mathrm{CDCl}_{3}\right): \delta 2.19\left(3 \mathrm{H}, \mathrm{s}, 4-\mathrm{CH}_{3}\right), 2.49\left(3 \mathrm{H}, \mathrm{s}, \mathrm{CH}_{3}\right), 3.73(2 \mathrm{H}$, s, $\left.\mathrm{CH}_{2}\right), 6.15(1 \mathrm{H}, \mathrm{s},-\mathrm{CH}=), 7.26-7.30(2 \mathrm{H}, \mathrm{m}, \mathrm{Ar}-\mathrm{H}), 7.36-7.39(3 \mathrm{H}, \mathrm{m}, \mathrm{Ar}-\mathrm{H}), 7.76-7.82(4 \mathrm{H}$, $\mathrm{m}, \mathrm{Ar}-\mathrm{H}), 12.93(1 \mathrm{H}, \mathrm{s}, \mathrm{NH}) .{ }^{13} \mathrm{C} \mathrm{NMR}\left(100 \mathrm{MHz}, \mathrm{CDCl}_{3}\right): \delta 21.6\left(4-\mathrm{CH}_{3}\right), 22.5\left(=\mathrm{C}-\underline{\mathrm{CH}}_{3}\right), 45.7$

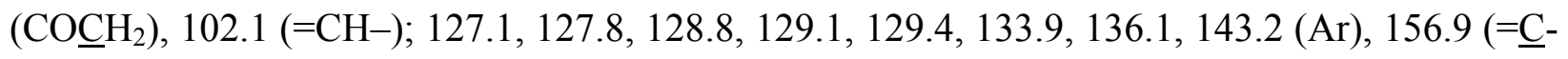


$\left.\mathrm{CH}_{3}\right), 170.7(\underline{\mathrm{COCH}} 2), 191.2$ (을); Anal. Calcd for: $\mathrm{C}_{19} \mathrm{H}_{19} \mathrm{NO}_{2} \mathrm{C}, 77.79 ; \mathrm{H}, 6.53 ; \mathrm{N}, 4.77$. Found: C, 77.83; H, 6.55; N, 4.80\%.

$\boldsymbol{N}$-[(Z)-3-(4-chlorophenyl)-1-methyl-3-oxoprop-1-en-1-yl]-2-phenylacetamide ( $Z$-3g). Yield $65 \%$, white crystals, mp $110-111^{\circ} \mathrm{C}$. IR $(\mathrm{KBr}): v, \mathrm{~cm}^{-1} 3455(\mathrm{NH}), 1708(\mathrm{C}=\mathrm{O}), 1618(\mathrm{C}=\mathrm{O})$, $\left.1594(\mathrm{~N}-\mathrm{C}=\mathrm{O}) .{ }^{1} \mathrm{H} \mathrm{NMR}\left(400 \mathrm{MHz}, \mathrm{CDCl}_{3}\right)\right): \delta 2.49\left(3 \mathrm{H}, \mathrm{s}, \mathrm{CH}_{3}\right), 3.74\left(2 \mathrm{H}, \mathrm{s}, \mathrm{CH}_{2}\right), 5.97(1 \mathrm{H}$, $\mathrm{s},-\mathrm{CH}=), 7.35-7.44(7 \mathrm{H}, \mathrm{m}, \mathrm{Ar}-\mathrm{H}), 7.79-7.83(2 \mathrm{H}, \mathrm{m}, \mathrm{Ar}-\mathrm{H}), 12.84(1 \mathrm{H}, \mathrm{s}, \mathrm{NH}) .{ }^{13} \mathrm{C}$ NMR $(100$ $\left.\left.\mathrm{MHz}, \mathrm{CDCl}_{3}\right)\right): \delta 22.6\left(=\mathrm{C}-\underline{\mathrm{CH}}_{3}\right), 45.7\left(\mathrm{COCH}_{2}\right), 101.7(=\mathrm{CH}-), 127.4,128.8,128.9,129.1$,

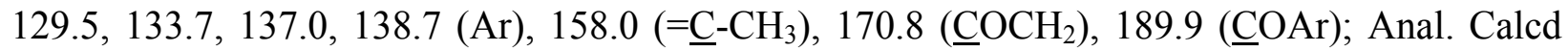
for: $\mathrm{C}_{18} \mathrm{H}_{16} \mathrm{ClNO}_{2} \mathrm{C}, 68.90 ; \mathrm{H}, 5.14 ; \mathrm{N}, 4.46$. Found: C, 68.94; H, 5.12; N, 4.51\%.

$\boldsymbol{N}$-[(Z)-1-methyl-3-(2-naphthyl)-3-oxoprop-1-en-1-yl]-2-phenylacetamide $(\boldsymbol{Z}-3 \mathrm{~h})$. Yield 70\%, white crystals, mp 99-100 ${ }^{\circ} \mathrm{C}$. IR (KBr): $v, \mathrm{~cm}^{-1} 3460(\mathrm{NH}), 1710(\mathrm{C}=\mathrm{O}), 1620(\mathrm{~N}-\mathrm{C}=\mathrm{O}), 1598$ $(\mathrm{C}=\mathrm{C}) .{ }^{1} \mathrm{H}$ NMR $\left(400 \mathrm{MHz}, \mathrm{CDCl}_{3}\right): \delta 2.55\left(3 \mathrm{H}, \mathrm{s}, \mathrm{CH}_{3}\right), 3.77\left(2 \mathrm{H}, \mathrm{s}, \mathrm{CH}_{2}\right), 6.20(1 \mathrm{H}, \mathrm{s},-\mathrm{CH}=)$, 7.29-7.45 (5H, m, H Ar), 7.51-7.61 (2H, m, Ar-H), 7.85-8.00 (4H, m, Ar-H), 8.41 (1H, s, H-1' Ar), $12.97(1 \mathrm{H}, \mathrm{s}, \mathrm{NH}) .{ }^{13} \mathrm{C}$ NMR $\left(100 \mathrm{MHz}, \mathrm{CDCl}_{3}\right): \delta 22.5\left(=\mathrm{C}-\underline{\mathrm{CH}}_{3}\right), 45.7\left(\mathrm{COCH}_{2}\right), 102.3$ (=CH-), 123.9, 126.7, 127.4, 127.7, 128.1, 128.4, 128.8, 128.9, 129.4, 132.7, 133.9, 135.3, 136.1 (Ar), $157.3\left(=\underline{\mathrm{C}}-\mathrm{CH}_{3}\right), 170.7\left(\underline{\mathrm{COCH}_{2}}\right), 191.2(\mathrm{COAr}) ;$ Anal. Calcd for: $\mathrm{C}_{22} \mathrm{H}_{19} \mathrm{NO}_{2} \mathrm{C}, 80.22 ; \mathrm{H}$, $5.81 ; \mathrm{N}, 4.25$. Found: C, 80.25; H, 5.80; N, 4.19\%.

$\boldsymbol{N}$-[(Z)-1-methyl-3-(1-naphthyl)-3-oxoprop-1-en-1-yl]-2-phenylacetamide ( $Z$-3i). Yield 63\%, white crystals, mp 58-59 ${ }^{\circ} \mathrm{C}$. IR (KBr): $v, \mathrm{~cm}^{-1} 3455(\mathrm{NH}), 1708(\mathrm{C}=\mathrm{O}), 1618(\mathrm{C}=\mathrm{O}), 1594(\mathrm{~N}-$ $\mathrm{C}=\mathrm{O}) .{ }^{1} \mathrm{H}$ NMR $\left(400 \mathrm{MHz}, \mathrm{CDCl}_{3}\right): \delta 2.51\left(3 \mathrm{H}, \mathrm{s}, \mathrm{CH}_{3}\right), 3.80\left(2 \mathrm{H}, \mathrm{s}, \mathrm{CH}_{2}\right), 5.88(1 \mathrm{H}, \mathrm{s},-\mathrm{CH}=)$, 7.31-7.61 (8H, m, Ar-H), 7.67-7.71 (1H, m, Ar-H), 7.86-7.96 (2H, m, H Ar), 8.34-8.39 (1H, m, $\mathrm{Ar}-\mathrm{H}), 12.90(1 \mathrm{H}, \mathrm{s}, \mathrm{NH}) .{ }^{13} \mathrm{C} \mathrm{NMR}\left(100 \mathrm{MHz}, \mathrm{CDCl}_{3}\right): \delta 22.4\left(=\mathrm{C}-\underline{\mathrm{CH}}_{3}\right), 45.8\left(\mathrm{COCH}_{2}\right), 106.6$ $(=\mathrm{CH}-), 124.6,125.6,126.3,126.4,127.2,127.3,127.5,128.5,129.0,129.6,130.0,131.5$,

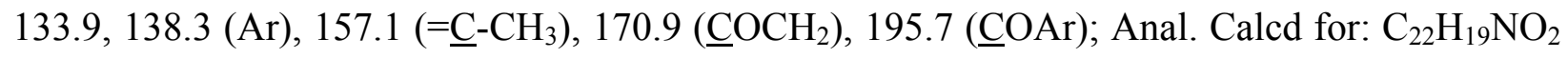
C, 80.22; H, 5.81; N, 4.25. Found: C, 80.18; H, 5.82; N, 4.29\%.

$\boldsymbol{N}$-[2-oxo-cyclohex-(Z,E)-ylidenemethyl]-2-phenylacetamide $\quad(\boldsymbol{Z}, \boldsymbol{E}-\mathbf{3 k})$. Yield $39 \%$, lightyellow oil. Individual isomers were isolated by column chromatography (silicagel L 40/100, $\mathrm{CHCl}_{3}$-EtOAc). Z-3k. White crystals, $\mathrm{mp} 85-86{ }^{\circ} \mathrm{C}$ (hexane). IR $\left(\mathrm{CHCl}_{3}\right): v, \mathrm{~cm}^{-1} 3248(\mathrm{NH})$, $1698(\mathrm{C}=\mathrm{O}), 1655(\mathrm{C}=\mathrm{O}), 1583(\mathrm{~N}-\mathrm{C}=\mathrm{O}) .{ }^{1} \mathrm{H}$ NMR $\left(400 \mathrm{MHz}, \mathrm{CDCl}_{3}\right) \quad$ ): $\delta 1.63-1.80,2.31-$ $2.45\left(8 \mathrm{H}, \mathrm{m},\left(\mathrm{CH}_{2}\right)_{4}\right), 3.67\left(2 \mathrm{H}, \mathrm{s}, \mathrm{CH}_{2}\right), 7.22(1 \mathrm{H}, \mathrm{dt}, J 10.7,1.5 \mathrm{~Hz},=\mathrm{CH}), 7.28-7.42(5 \mathrm{H}, \mathrm{m}$, $\left.\mathrm{C}_{6} \mathrm{H}_{5}\right), 11.32(1 \mathrm{H}, \mathrm{m}, \mathrm{NH}) .{ }^{13} \mathrm{C} \mathrm{NMR}\left(100 \mathrm{MHz}, \mathrm{CDCl}_{3}\right): \delta 22.4,23.3,28.8,39.4\left(\mathrm{CH}_{2}\right)_{4}, 44.1$ $\left(\mathrm{C}_{6} \mathrm{H}_{5}-\underline{\mathrm{CH}}_{2}\right), 113.5$ (=드), 127.7, 129.1, 129.4, $133.5\left(\mathrm{C}_{6} \mathrm{H}_{5}\right), 133.9(=\mathrm{CHN}), 170.1(\mathrm{NHCO})$, $203.7(\mathrm{C}=\mathrm{O})$. Anal. Calcd for: $\mathrm{C}_{15} \mathrm{H}_{17} \mathrm{NO}_{2} \mathrm{C}$, 74.05; H, 7.04; N, 5.76. Found: C, 73.93; H, 7.06; N, 5.77\%. E-3k. White crystals, mp 137-138 ${ }^{\circ} \mathrm{C}$ (EtOAc); IR $\left(\mathrm{CHCl}_{3}\right): v, \mathrm{~cm}^{-1} 3412(\mathrm{NH}), 1712$ $(\mathrm{C}=\mathrm{O}), 1670(\mathrm{C}=\mathrm{O}), 1551(\mathrm{~N}-\mathrm{C}=\mathrm{O}) .{ }^{1} \mathrm{H}$ NMR (400 MHz, $\left.\mathrm{CDCl}_{3}\right): \delta$ 1.62-1.80, 2.01-2.07, 2.32-2.38 (8H, m, $\left.\left(\mathrm{CH}_{2}\right)_{4}\right), 3.72\left(2 \mathrm{H}, \mathrm{s}, \mathrm{CH}_{2}\right), 7.13(1 \mathrm{H}, \mathrm{d}, J 12,1 \mathrm{~Hz}, \mathrm{NH}), 7.25-7.42(5 \mathrm{H}, \mathrm{m}$, $\left.\mathrm{C}_{6} \mathrm{H}_{5}\right), 7.94(1 \mathrm{H}, \mathrm{dt}, J 12.1,2.0 \mathrm{~Hz},=\mathrm{CH}) .{ }^{13} \mathrm{C} \mathrm{NMR}\left(100 \mathrm{MHz}, \mathrm{CDCl}_{3}\right): \delta 22.5,22.6,24.0,39.5$ $\left(\mathrm{CH}_{2}\right)_{4}, 43.9\left(\mathrm{C}_{6} \mathrm{H}_{5}-\underline{C H}_{2}\right), 116.5$ (= $\left.\underline{\mathrm{CCO}}\right), 128.0(=\mathrm{CHN}), 129.1,129.4,129.7,133.5\left(\mathrm{C}_{6} \mathrm{H}_{5}\right)$, 168.6 (NHCO), $199.0(\mathrm{C}=\mathrm{O})$; Anal. Calcd for: $\mathrm{C}_{15} \mathrm{H}_{17} \mathrm{NO}_{2} \mathrm{C}, 74.05$; H, 7.04; N, 5.76. Found: C, $73.94 ; \mathrm{H}, 7.04 ; \mathrm{N}, 5.76 \%$. 
1,4,6-Trimethyl-3-phenyl-1H-pyridin-2-one (7a). Powdered $\mathrm{KOH} 0.112 \mathrm{~g}$ (1.5 mmol) was added to a solution of compound Z-3a $0.232 \mathrm{~g}(1.0 \mathrm{mmol})$ in EtOH. The mixture was stirred for $15 \mathrm{~min}$ and then $\mathrm{H}_{2} \mathrm{O}(10 \mathrm{~mL})$ added, evaporated to $2 / 3$, and cooled. The solid product was filtered off, and recrystallized from a mixture of solvents: $\mathrm{EtOH}-\mathrm{H}_{2} \mathrm{O}$ yielding $0.20 \mathrm{~g}(94 \%)$ of product with mp 118-119 ${ }^{\circ} \mathrm{C}$. IR (KBr): $v, \mathrm{~cm}^{-1} 1642(\mathrm{NC}=\mathrm{O}) .{ }^{1} \mathrm{H}$ NMR $\left(400 \mathrm{MHz}, \mathrm{CDCl}_{3}\right): \delta$ $1.99\left(3 \mathrm{H}, \mathrm{s}, 4-\mathrm{CH}_{3}\right), 2.35\left(3 \mathrm{H}, \mathrm{s}, 6-\mathrm{CH}_{3}\right), 3.53\left(3 \mathrm{H}, \mathrm{s}, 1-\mathrm{CH}_{3}\right), 6.01(1 \mathrm{H}, \mathrm{s}, \mathrm{H}-5), 7.22-7.42(5 \mathrm{H}$, m, $\left.\mathrm{C}_{6} \mathrm{H}_{5}\right) .{ }^{13} \mathrm{C}$ NMR (100 MHz, $\left.\mathrm{CDCl}_{3}\right): \delta 20.3\left(6-\mathrm{CH}_{3}\right), 20.8\left(4-\mathrm{CH}_{3}\right), 31.4\left(1-\mathrm{CH}_{3}\right), 109.4(\mathrm{C}-$ 5), $127.0\left(\mathrm{C}_{6} \mathrm{H}_{5}, \mathrm{C}-4^{\prime}\right), 127.8$ (C-3), $128.1\left(\mathrm{C}_{6} \mathrm{H}_{5}, \mathrm{C}-3^{\prime}, \mathrm{C}-5^{\prime}\right), 130.1\left(\mathrm{C}_{6} \mathrm{H}_{5}, \mathrm{C}-2^{\prime}, \mathrm{C}-6^{\prime}\right), 136.8$ $\left(\mathrm{C}_{6} \mathrm{H}_{5}, \mathrm{C}-1^{\prime}\right), 143.5$ (C-6), 146.0 (C-4), 163.0 (C-2); m/z 213 [M] ${ }^{+\cdot}$; Anal. Calcd for: $\mathrm{C}_{14} \mathrm{H}_{15} \mathrm{NO}$ C, 78.84; H, 7.09; N, 6.57. Found: C, 78.89; H, 7.13; N, 6.54\%.

General procedure for the synthesis of 3-phenylpyridin-2(1H)-one (7b-i). $\mathrm{KO} t$ - $\mathrm{Bu}(0.084 \mathrm{~g}$, $0.75 \mathrm{mmol})$ was added with ice-cooling and stirring to a solution of phenylacetamide $3(0.5$ $\mathrm{mmol}$ ) in absolute THF (4 ml). After 5-18 h (monitored by TLC) the solvent was evaporated, the residue triturated with $\mathrm{H}_{2} \mathrm{O}$ and then neutralized with aq 5\% AcOH solution. The product was filtered off, and recrystallized from $\mathrm{MeOH}(\mathbf{7 g})$, EtOH (7b-e) or $i-\mathrm{PrOH}(\mathbf{7 f}, \mathbf{h}, \mathbf{i})$.

4-Methyl-3-phenyl-5,6,7,8-tetrahydro-1H-quinolin-2-one (7c). The mixture was stirred for 5 hours. Yield $62 \%$, white crystals, mp above $280{ }^{\circ} \mathrm{C}$. IR $(\mathrm{KBr}): v, \mathrm{~cm}^{-1} 1631(\mathrm{NC}=\mathrm{O}), 3278(\mathrm{NH})$. ${ }^{1} \mathrm{H}$ NMR (400 MHz, $\left.\mathrm{CDCl}_{3}\right): \delta 1.63-1.86(4 \mathrm{H}, \mathrm{m}, 2 \mathrm{H}-6,2 \mathrm{H}-7), 1.93\left(3 \mathrm{H}, \mathrm{s}, \mathrm{CH}_{3}\right), 2.35-2-2.61$ (4H, m, 2H-5, 2H-8), 7.18-7.34 (3H, m, $\left.\mathrm{C}_{6} \mathrm{H}_{5}\right), 7.35-7.45\left(2 \mathrm{H}, \mathrm{m}, \mathrm{C}_{6} \mathrm{H}_{5}\right), 12.12$ (1H, br. s, NH). ${ }^{13} \mathrm{C} \mathrm{NMR}\left(100 \mathrm{MHz}, \mathrm{CDCl}_{3}\right): \delta 17.3\left(4-\mathrm{CH}_{3}\right), 21.6,22.8,24.6,27.1\left(4 \mathrm{CH}_{2}\right), 114.1(\mathrm{C}-4), 126.8$, 128.0, 130.4, 136.8, $136.8\left(\mathrm{C}_{6} \mathrm{H}_{5}\right), 128.3$ (C-3), 140.9 (C-6), 149.1 (C-4), 162.8 (C-2); m/z 239 $[\mathrm{M}]^{+\cdot}$. Anal. Calcd for: $\mathrm{C}_{16} \mathrm{H}_{17} \mathrm{NO} \mathrm{C}, 80.30 ; \mathrm{H}, 7.16 ; \mathrm{N}, 5.85$. Found: C, 80.38; H, 7.22; N, $5.91 \%$.

1-Methyl-4-phenyl-2,5,6,7-tetrahydro-3H-cyclopenta[c]pyrindin-3-one (7d). The reaction mixture was stirred for $7 \mathrm{~h}$. Yield $40 \%$, white crystals, $\mathrm{mp}>280{ }^{\circ} \mathrm{C}$. IR (KBr): $v, \mathrm{~cm}^{-1} 1640$ $(\mathrm{NC}=\mathrm{O}), 3276(\mathrm{NH}) .{ }^{1} \mathrm{H} \mathrm{NMR}\left(400 \mathrm{MHz}, \mathrm{CDCl}_{3}\right): \delta 1.97\left(2 \mathrm{H}, \mathrm{tt}(\mathrm{q}), J 7.2,7.3 \mathrm{~Hz}, \mathrm{CH}_{2}\right), 2.26$ $\left(3 \mathrm{H}, \mathrm{s}, \mathrm{CH}_{3}\right), 2.69\left(2 \mathrm{H}, \mathrm{t}, J 7.2 \mathrm{~Hz}, \mathrm{CH}_{2}\right), 2.78\left(2 \mathrm{H}, \mathrm{t}, J 7.3 \mathrm{~Hz}, \mathrm{CH}_{2}\right), 7.24-7.34,7.35-7.53(5 \mathrm{H}$, $\left.\mathrm{m}, \mathrm{C}_{6} \mathrm{H}_{5}\right), 12.78\left(1 \mathrm{H}\right.$, br. s, NH). ${ }^{13} \mathrm{C} \mathrm{NMR}\left(100 \mathrm{MHz}, \mathrm{CDCl}_{3}\right): \delta 16.8\left(6-\mathrm{CH}_{3}\right), 25.6,28.7,33.5$ $\left(3 \mathrm{CH}_{2}\right), 121.2(\mathrm{C}-5), 123.0(\mathrm{C}-3), 126.8,127.8,129.8,136.0\left(\mathrm{C}_{6} \mathrm{H}_{5}\right), 137.3(\mathrm{C}-6), 157.8(\mathrm{C}-4)$, 164.1 (C-2); $m / z 225[\mathrm{M}]^{+*}$. Anal. Calcd for: $\mathrm{C}_{15} \mathrm{H}_{15} \mathrm{NO} \mathrm{C}, 79.97 ; \mathrm{H}, 6.71 ; \mathrm{N}, 6.22$. Found: $\mathrm{C}$, $80.04 ; \mathrm{H}, 6.82 ; \mathrm{N}, 6.27 \%$.

6-Methyl-3,4-diphenylpyridin-2(1H)-one (7e). The reaction mixture was stirred for $7 \mathrm{~h}$.Yield 70\%, white crystals, mp 228-229 C; IR (KBr): $v, \mathrm{~cm}^{-1} 3476,2787(\mathrm{NH}), 1628(\mathrm{~N}-\mathrm{C}=\mathrm{O}) .{ }^{1} \mathrm{H}$ NMR (400 MHz, DMSO-d $\left.d_{6}\right): \delta 2.32\left(3 \mathrm{H}, \mathrm{s}, 6-\mathrm{CH}_{3}\right), 6.13(1 \mathrm{H}, \mathrm{s}, \mathrm{H}-5), 7.00-7.24(10 \mathrm{H}, \mathrm{m}, \mathrm{H}$ Ar), 12.86 (1H, br.s, NH). ${ }^{13} \mathrm{C}$ NMR (100 MHz, DMSO- $\left.d_{6}\right): \delta 18.9\left(6-\mathrm{CH}_{3}\right), 108.7(\mathrm{C}-5), 126.6$ (C-3), 126.1, 127.5, 131.3, 135.4, 127.5, 127.9, 128.9, 139.7 (Ar), 143.7 (C-6), 152.2 (C-4), 164.7 (C-2); Anal. Calcd for: $\mathrm{C}_{18} \mathrm{H}_{15} \mathrm{NO}$ C, 82.73; H, 5.79; N, 5.36. Found: C, 82.76; H, 5.80; N, $5.42 \%$. 
6-Methyl-4-(4-methylphenyl)-3-phenylpyridin-2(1H)-one (7f). The reaction mixture was stirred for 10 h.Yield $62 \%$, light crystals, mp $275^{\circ} \mathrm{C}$ (decomp.); IR (KBr): $v, \mathrm{~cm}^{-1} 3453,2767$ $(\mathrm{NH}), 1621(\mathrm{~N}-\mathrm{C}=\mathrm{O}) .{ }^{1} \mathrm{H}$ NMR $\left(400 \mathrm{MHz}, \mathrm{DMSO}-d_{6}\right): \delta 2.20\left(3 \mathrm{H}, \mathrm{s}, 4^{\prime}-\mathrm{CH}_{3}\right), 2.21(3 \mathrm{H}, \mathrm{s}, 6-$ $\mathrm{CH}_{3}$ ), 6.02 (1H, s, H-5), 6.90-7.02 (6H, m, H Ar), 7.09-7.17 (3H, m, H Ar), 11.75 (1H, br.s, $\mathrm{NH}) .{ }^{13} \mathrm{C}$ NMR (100 MHz, DMSO- $\left.d_{6}\right): \delta 18.9\left(6-\mathrm{CH}_{3}\right), 21.1\left(4^{\prime}-\mathrm{CH}_{3}\right), 107.2(\mathrm{C}-5), 126.7(\mathrm{C}-3)$, 125.90, 127.7, 129.0, 129.1, 131.6, 136.7, 137.1, 137.2 (Ar), 144.2 (C-6), 150.8 (C-4), 162.9 (C2); Anal. Calcd for: $\mathrm{C}_{19} \mathrm{H}_{17} \mathrm{NO}$ C, 82.88; H, 6.22; N, 5.09. Found: C, 82.90; H, 6.25; N, 5.13\%.

4-(4-Chlorophenyl)-6-methyl-3-phenylpyridin-2(1H)-one $\mathbf{( 7 g )}$. The reaction mixture was stirred for $7 \mathrm{~h}$. Yield $73 \%$, light crystals, mp $287{ }^{\circ} \mathrm{C}$ (decomp.); IR (KBr): $v, \mathrm{~cm}^{-1} 3451,2765$ $(\mathrm{NH}), 1622(\mathrm{~N}-\mathrm{C}=\mathrm{O}) .{ }^{1} \mathrm{H}$ NMR $\left(400 \mathrm{MHz}, \mathrm{DMSO}-d_{6}\right): \delta 2.21\left(3 \mathrm{H}, \mathrm{s}, 6-\mathrm{CH}_{3}\right), 6.04(1 \mathrm{H}, \mathrm{s}, \mathrm{H}-5)$, 6.98-7.07 (4H, m, H Ar), 7.11-7.19 (3H, m, H Ar), 7.22-7.27 (2H, m, H Ar), 11.85 (1H, br.s, $\mathrm{NH}) .{ }^{13} \mathrm{C}$ NMR $\left(100 \mathrm{MHz}, \mathrm{DMSO}-d_{6}\right): \delta 18.9\left(6-\mathrm{CH}_{3}\right), 106.9$ (C-5), $126.9(\mathrm{C}-3), 126.3,127.9$, 128.5, 131.0, 131.5, 132.7, 136.2, 138.8 (Ar), 144.7 (C-6), 149.7 (C-4), 162.8 (C-2); Anal. Calcd for: $\mathrm{C}_{18} \mathrm{H}_{14} \mathrm{ClNO}$ C, 73.10; H, 4.77; N, 4.74. Found: C, 73.06; H, 4.80; N, 4.79\%.

6-Methyl-4-(2-naphthyl)-3-phenylpyridin-2(1H)-one $(7 \mathrm{~h})$. The reaction mixture was stirred for $18 \mathrm{~h}$. Yield $65 \%$, light crystals, mp $260{ }^{\circ} \mathrm{C}$ (decomp.); IR (KBr): $v, \mathrm{~cm}^{-1} 3440,2775(\mathrm{NH})$, $1624(\mathrm{~N}-\mathrm{C}=\mathrm{O}) .{ }^{1} \mathrm{H}$ NMR $\left(400 \mathrm{MHz}, \mathrm{DMSO}-d_{6}\right): \delta 2.25\left(3 \mathrm{H}, \mathrm{s}, 6-\mathrm{CH}_{3}\right), 6.17(1 \mathrm{H}, \mathrm{s}, \mathrm{H}-5), 7.03-$ 7.13 (6H, m, H Ar), 7.43-7.48 (2H, m, H Ar), 7.65 (1H, s, H-1' Ar), 7.72-7.81 (3H, m, H Ar), $11.74\left(1 \mathrm{H}\right.$, br.s, NH). ${ }^{13} \mathrm{C}$ NMR $\left(100 \mathrm{MHz}, \mathrm{DMSO}-d_{6}\right): \delta 18.9\left(6-\mathrm{CH}_{3}\right), 107.4(\mathrm{C}-5), 126.7(\mathrm{C}-$ 3), 126.7, 126.8, 127.2, 127.5, 127.7, 127.9, 128.1, 128.4, 131.6, 132.4, 133.4, 133.0, 136.5, 137.6 (Ar), 144.5 (C-6), 150.8 (C-4), 162.9 (C-2); Anal. Calcd for: $\mathrm{C}_{22} \mathrm{H}_{17} \mathrm{NO}$ C, 84.86; H, 5.50; $\mathrm{N}, 4.50$. Found: C, 84.87; H, 5.52; N, 4.43\%.

6-Methyl-4-(1-naphthyl)-3-phenylpyridin-2(1H)-one (7i). The reaction mixture was stirred for 18 h. Yield 60\%, light crystals, mp $235^{\circ} \mathrm{C}$ (decomp.). IR (KBr): v, $\mathrm{cm}^{-1} 3429,2768(\mathrm{NH}), 1628$ $(\mathrm{N}-\mathrm{C}=\mathrm{O}) .{ }^{1} \mathrm{H}$ NMR $\left(400 \mathrm{MHz}, \mathrm{DMSO}-d_{6}\right): \delta 2.22\left(3 \mathrm{H}, \mathrm{s}, 6-\mathrm{CH}_{3}\right), 6.05(1 \mathrm{H}, \mathrm{s}, \mathrm{H}-5), 6.97-7.720$ (12H, m, H Ar), 11.82 (1H, br.s, NH). ${ }^{13} \mathrm{C}$ NMR (100 MHz, DMSO- $\left.d_{6}\right): \delta 18.9\left(6-\mathrm{CH}_{3}\right), 107.2$ (C-5), 126.7 (C-3), 126.1, 127.7, 128.4, 129.1, 131.6, 136.5140 .0 (Ar), 144.4 (C-6), 150.9 (C-4), 162.9 (C-2); Anal. Calcd for: $\mathrm{C}_{22} \mathrm{H}_{17} \mathrm{NO}$ C, 84.86; H, 5.50; N, 4.50. Found: C, 84.90; H, 5.55; N, $4.58 \%$.

\section{Acknowledgements}

This work was supported by the Russian Foundation for Basic Research (project 11-03-00338-a) and Ministry of Education and Science of the Russian Federation (project 2597). 


\section{References}

1. Nagarajan, M.; Xiao, X. S.; Antony, S.; Kohlhagen, G.; Pommier, Y.; Cushman, M. J. Med. Chem. 2003, 46, 5712-5724.

http://dx.doi.org/10.1021/jm030313f

2. Jayasinghe, L.; Abbas, H. K.; Jacob, M. R.; Herath, W. H. M. W.; Nanayakkara, N. P. D. J. Nat. Prod. 2006, 69, 439-442.

http://dx.doi.org/10.1021/np050487v

3. Houllier, N.; Gopisetti, J.; Lestage, P.; Lasne, M.-C.; Rouden, J. Bioorg. Med. Chem. Lett. 2010, 20, 6667-6670.

http://dx.doi.org/10.1016/j.bmcl.2010.09.017

4. Jessen, H. J.; Gademann, K. Nat. Prod. Rep. 2010, 27, 1168-1185.

http://dx.doi.org/10.1039/B911516C

5. Jessen, H. J.; Schumacher, A.; Shaw, T.; Pfaltz, A.; Gademann, K. Angew. Chem. Int. Ed. 2011, 50, $4222-4226$.

http://dx.doi.org/10.1002/anie.201007671

6. Wall, M. E.; Wani, M. C.; Cook, C. E.; Palmer, K. H.; McPhail, A. T.; Sim, G. A. J. Am. Chem. Soc. 1966, 88, 3888-3890.

http://dx.doi.org/10.1021/ja00968a057

7. Devert, M.; Sabot, C.; Giboreau, P.; Constant, J.-F.; Greene, A. E.; Kanazawa, A. Tetrahedron 2010, $66,7227-7231$.

http://dx.doi.org/10.1016/j.tet.2010.06.003

8. Odan, M.; Ishizuka, N.; Hiramatsu, Y.; Inagaki, M.; Hashizume, H.; Fujii, Y.; Mitsumori, S.; Morioka, Y.; Soga, M.; Deguchi, M.; Yasui, K.; Arimura, A. Bioorg. Med. Chem. Lett. 2012, 22, 2898-2901.

http://dx.doi.org/10.1016/j.bmcl.2012.02.050

9. Lizarzaburu, M.; Turcotte, S.; Du, X.; Duquette, J.; Fu, A.; Houze, J.; Li, L.; Liu, J.; Murakoshi, M.; Oda, K.; Okuyama, R.; Nara, F.; Reagan, J.; Yu, M.; Medina, J. C. Bioorg. Med. Chem. Lett. 2012, $22,5942-5947$.

http://dx.doi.org/10.1016/j.bmcl.2012.07.063

10. Bengtsson, Ch.; Lindgren, A. E. G.; Uvell, H.; Almqvist, F. Eur. J. Med. Chem. 2012, 54, 637-646. http://dx.doi.org/10.1016/j.ejmech.2012.06.018

11. Tilley, J. W.; Sidduri, A.; Lou, J.; Kaplan, G.; Tare, N.; Cavallo, G.; Frank, K.; Pamidimukkala, A.; Choi, D. S.; Gerber, L.; Railkar, A.; Renzetti, L. Bioorg. Med. Chem. Lett. 2013, 23, 1036-1040. http://dx.doi.org/10.1016/j.bmcl.2012.12.019

12. Heravi, M. M.; Hamidi H. J. Iran. Chem. Soc. 2013, 10, 265-273. http://dx.doi.org/10.1007/s13738-012-0155-7

13. Torres, M.; Gil, S.; Parra, M. Curr. Org. Chem. 2005, 9, 1757-1779. http://dx.doi.org/10.2174/138527205774610886 
14. Harker, W. R. R.; Delaney, P. M.; Simms, M.; Tozer, M. J.; Harrity, J. P. A. Tetrahedron 2013, 69, $1546-1552$

http://dx.doi.org/10.1016/j.tet.2012.12.010

15. Wang, X.-W.; Cui, H.-F.; Wang, H.-F.; Yang, Y.-Q.; Zhao, G.; Zhu, Sh.-Z. Tetrahedron 2011, 67, $2468-2473$.

http://dx.doi.org/10.1016/j.tet.2011.01.047

16. Zhou, Q.; Chu, X.; Tang, W.; Lu, T. Tetrahedron 2012, 68, 4152-4158. http://dx.doi.org/10.1016/j.tet.2012.03.106

17. Pflum, D. A. Camps Quinolinol Synthesis, In Name Reactions in Heterocyclic Chemistry, Li, J. J., Ed.; John Wiley \& Sons: New York, NY, 2005, 386-389.

18. Camps, R. Ber. 1899, 22, 3228-3234. http://dx.doi.org/10.1002/cber.18990320389

19. Camps, R. Arch. Pharm. 1899, 237, 659-691. http://dx.doi.org/10.1002/ardp.18992370902

20. Anderson, K., Buchwald, S. L. J. Org. Chem. 2007, 72, 7968-7973. http://dx.doi.org/10.1021/jo701384n

21. Mochalov, S. S.; Chasanov, M. I. Chem. Heterocycl. Compd. 2008, 44, 628-629. http://dx.doi.org/10.1007/s10593-008-0086-5

22. Mochalov, S. S.; Chasanov, M. I.; Fedotov, A. N.; Zefirov, N. S. Chem. Heterocycl. Compd. 2011, 47, 1105-1121.

http://dx.doi.org/10.1007/s10593-011-0881-2

23. Fisyuk, A. S.; Vorontsova, M. A.; Ivanov, S. A. Chem. Heterocycl. Compd. 1994, 30, 709-712 [Khim. Geterotsikl. Soedin. 1994, 812- 815]. http://dx.doi.org/10.1007/BF01166313

24. Fissyuk, A. S.; Vorontsova, M. A.; Temnikov, D. V.; Tetrahedron Lett. 1996, 37, 5203-5206. http://dx.doi.org/10.1016/0040-4039(96)01051-9

25. Fisyuk, A. S.; Berdovich, L. V.; Temnikov, D. V.; Knyaz'kova, L. N. Chem. Heterocycl. Compd. 1997, 33, 805-810 [Khim. Geterotsikl. Soedin. 1997, 921- 927].

http://dx.doi.org/10.1007/BF02253030

26. Fisyuk, A. S.; Vorontsova, M. A. Chem. Heterocycl. Compd. 1998, 34, 195-199 [Khim. Geterotsikl. Soedin. 1998, 220-224]. http://dx.doi.org/10.1007/BF02315183

27. Fisyuk, A. S.; Poendaev, N. V.; Bundel, Y. G. Mendeleev Commun. 1998, 8, 12-13. http://dx.doi.org/10.1070/MC1998v008n01ABEH000877

28. Fisyuk, A. S.; Poendaev, N. V. Molecules 2002, 7, 124-128. http://dx.doi.org/10.3390/70200124

29. Fisyuk, A. S.; Poendaev, N. V.; Molecules 2002, 7, 119-123. http://dx.doi.org/10.3390/70200119

30. Fisyuk, A. S., Poendaev, N. V. Chem. Heterocycl. Compd. 2003, 39, 895-900 [Khim. Geterotsikl. Soedin. 2003, 1037-1042]. 
http://dx.doi.org/10.1023/A:1026146421293

31. Wick, A. E.; Bartlett, P. A.; Dolphin D. Helv. Chim. Acta 1971, 54, 513-522.

http://dx.doi.org/10.1002/hlca.19710540210

32. Gewald, K.; Rehwald, M.; Müller, H.; Bellmann, P. Leibigs Ann. 1995, 5, 787-789. http://dx.doi.org/10.1002/jlac.1995199505115

33. Fisyuk, A. S.; Bogza, Y. P.; Poendaev, N. V.; Goncharov, D. S. Chem. Heterocycl. Compd. 2010, 46, 844-849 [Khim. Geterotsikl. Soedin. 2010 ,1044-1049].

http://dx.doi.org/10.1007/s10593-010-0592-0

34. Goncharov, D. S.; Kostuchenko, A. S.; Fisyuk, A. S. Chem. Heterocycl. Compd. 2009, 45, 793-795 [Khim. Geterotsikl. Soedin. 2009, 1005-1007].

http://dx.doi.org/10.1007/s10593-009-0358-8

35. Hommes, P.; Berlin, S.; Reissig, H.-U. Synthesis. 2013, 45, 3288-3294.

http://dx.doi.org/10.1055/s-0033-1338548

36. Fisyuk, A. S.; Kulakov I. V.; Goncharov, D. S. Nikitina O. S., Bogza, Y. P.; Shatsauskas A. L. Chem. Heterocycl. Compd. 2014, 50, 217-224 [Khim. Geterotsikl. Soedin. 2014, 241-249].

http://dx.doi.org/10.1007/s10593-014-1464-9

37. Shabana, R.; Rasmussen, J. B.; Lawesson, S.-O. Tetrahedron 1981, 37, 1819-1822.

http://dx.doi.org/10.1016/S0040-4020(01)98950-1

38. Kania, L.; Kamienska-Triela, K.; Witanowski, M. J. Mol. Struct. 1983, 102, 1-17. http://dx.doi.org/10.1016/0022-2860(83)80001-5

39. Henry, F.; Holtzclaw, H. F., Jr.; Collman, J. P.; Alire, R. M. J. Am. Chem. Soc. 1958, 80, 1100-1103. http://dx.doi.org/10.1021/ja01538a021

40. Baraldi, P. G. Simoni, D. Manfredini, S. Synthesis 1983, 902-903. http://dx.doi.org/10.1055/s-1983-30557

41. Valduga, C. J.; Squizani, A.; Braibante, H. S.; Braibante, M. E. F. Synthesis 1998, 1019-1022. http://dx.doi.org/10.1055/s-1998-2107

42. Singh, B. Lesher, G. Y. J. Heterocyclic. Chem. 1990, 27, 2085-2091. http://dx.doi.org/10.1002/jhet.5570270743

43. Thummel, R. P.; Kohli, D. K. J. Org. Chem. 1977, 42, $2742-2747$. http://dx.doi.org/10.1021/jo00436a019 